\title{
microRNA-199a-3p functions as tumor suppressor by regulating glucose metabolism in testicular germ cell tumors
}

\author{
XIAOWEN LIU ${ }^{1 *}$, HONGYAN DUAN ${ }^{1 *}$, SHIHUA ZHOU $^{1}$, ZHIYONG LIU $^{1}$, \\ DAOBING WU ${ }^{1}$, TING ZHAO ${ }^{1}$, SHAN XU $^{2}$, LIFANG YANG $^{2}$ and DAN LI ${ }^{1}$ \\ ${ }^{1}$ Department of Life Science, College of Biology, Hunan University, Changsha, Hunan 410082; ${ }^{2}$ Cancer Research Institute, \\ Xiangya School of Medicine, Central South University, Changsha, Hunan 410078, P.R. China
}

Received May 19, 2015; Accepted May 25, 2016

DOI: $10.3892 / \mathrm{mmr} .2016 .5472$

\begin{abstract}
RNA (miR)-199a-3p serves critical roles in cancer development and progression. In order to improve knowledge of the functional mechanism of miR-199a-3p in testicular tumors, the present study characterized the regulation of aerobic glycolysis by miR-199a-3p and its impact on metabolism. Using 3-4,5-dimethylthiazol-2-yl-2,5 diphenyl tetrazolium bromide, wound healing and flow cytometry assays, it was determined that overexpression of miR-199a-3p in Ntera- 2 cells caused suppression of cell growth and migration. Further biochemical methods and high-throughput quantitative polymerase chain reaction array of metabolic genes showed that inhibition of miR-199a-3p markedly elevated lactate production and 12 differentially expressed genes, including 2 upregulated and 10 downregulated genes, were identified following treatment with miR-199a-3p in Ntera-2 cells. In clinical samples, four selected genes, lactate dehydrogenase A, monocarboxylate transporter 1, phosphoglycerate kinase 1 and TP53-inducible glycolysis and apoptosis regulator, were significantly overexpressed in malignant testicular germ cell tumor, and their expression inversely correlated with the expression of miR-199a-3p, suggesting that these four genes may be affected by miR-199a-3p. Using bioinformatics analysis, the transcription factor $\mathrm{Sp} 1$ binding site was identified in the promoter region of the four selected genes. In addition, miR-199a-3p was predicted to bind to
\end{abstract}

Correspondence to: Dr Dan Li, Department of Life Science, College of Biology, Hunan University, 1 Denggao Road, Yuelu Mountain, Changsha, Hunan 410082, P.R. China

E-mail: lidanie@hotmail.com

Dr Lifang Yang, Cancer Research Institute, Xiangya School of Medicine, Central South University, 110 Xiangya Road, Changsha, Hunan 410078, P.R. China

E-mail: yanglifang99@ hotmail.com

${ }^{*}$ Contributed equally

Key words: microRNA-199a-3p, tumor suppressor, metabolism of glucose, testicular germ cell tumors conservative target sequences in the 3'-untranslated region of Sp1 mRNA, suggesting that miR-199a-3p may downregulate these four metabolic genes through $\mathrm{Sp1}$. It was demonstrated the dysregulated expression and activation of miR-199a-3p may serve important roles in aerobic glycolysis and tumorigenesis in patients with testicular cancer. Therefore, miR-199a-3p may be a potential biomarker in the prognosis and treatment of testicular tumors.

\section{Introduction}

micro (mi)RNAs are endogenous, small and non-coding RNAs, which negatively regulate protein expression by mRNA cleavage or translation repression (1). Since the first miRNA was reported in Caenorhabditis elegans by Lee et al (2), an large quantity of miRNAs have been identified and subsequent studies indicated that they serve crucial roles in various biological process and regulate the expression of up to $30 \%$ of human genes $(3,4)$. Previous studies suggested that some specific miRNAs have an important role in the pathogenesis of tumors by functioning as oncogenes or tumor suppressor genes (5-7). In addition, expression profiling of miRNAs can also be used for differentiation of the major histological subtypes of tumors $(8,9)$. As a result of their high sensitivity and the relatively easy method of detection, miRNAs have been shown to exhibit great potential as novel biomarkers for diagnosis, prognosis and therapy in cancer (9).

Testicular germ cell tumors (TGCTs) arise from carcinoma in situ cells, which resemble malignant primordial germ cells of fetal origin. Histologically, TGCTs are divided into seminomas and non-seminomas. For the clinical management of TGCTs, blood-based markers, including lactate dehydrogenase, $\alpha$-fetoprotein and human chorionic gonadotropin are used for diagnosis, risk assessment and determining patient prognosis (9). However, these biomarkers are useless for $\sim 40 \%$ of patients with TGCTs, suggesting that it is necessary to discover novel candidate biomarkers. Recently, certain miRNAs, including miR-372, $-373,-449,-383$ and -199 a have been identified to be dysregulated in TGCTs and they contribute to the development of TGCTs (9-11). Among them, miR-199a-3p, one of the miR-199a family, is of particular interest since it was reported to be specifically upregulated by transforming growth factor (TGF)- $\beta 1$ signaling in mouse GC-1 spg germ 
cells and may be associated with germ cell development in our previous study (12). In testicular cancer, miR-199a-3p has been reported to suppress cell growth, migration, invasion and metastasis by regulating TGF- $\beta 1$ signaling through the regulation of Smad4 (13). Chen et al (11) recently found that miR-199a-3p negatively regulated DNA methylation, partly through targeting DNA (cytosine-5)-methyltransferase 3A, in TGCTs (11). Although these interesting functional studies have addressed the role of miR-199a-3p in cancer development and progression, the regulatory role and underlying mechanisms of miR-199a-3p in metabolism of TGCTs remains unknown.

The present study characterized the regulation of aerobic glycolysis by miR-199a-3p and its impact on testicular cancer metabolism. It was revealed that miR-199a-3p suppressed cell growth and migration, and inhibition of miR-199a-3p increased the production of lactate in Ntera- 2 cells and affected testicular tumor metabolism by downregulating metabolic genes, including lactate dehydrogenase A (LDHA), monocarboxylate transporter 1 (MCT1), phosphoglycerate kinase 1 (PGK1) and TP53-inducible glycolysis and apoptosis regulator (TIGAR), suggesting that miR-199a-3p functions as a tumor suppressor in TGCTs by regulating the metabolism signaling pathway.

\section{Materials and methods}

Clinical tissue specimens. Formalin fixed paraffin embedded (FFPE) human testicular tumors tissue specimens, including adjacent non-tumor tissues, were collected at the Department of Pathology, Xiangya Hospital of Central South University (Hunan, China). Clinical tissue specimens included five normal testes and five TGCTs tissues. The present study was approved by the Independent Ethical Committee of Xiangya Hospital of Central South University.

Cell culture and transient transfections. The human Ntera-2 testicular tumor cell line [CRL-1973; American Type Culture Collection (ATCC), Rockville, MD, USA] and the human embryonic kidney cell line HEK293 (CRL-1573; ATCC) were cultured in RPMI-1640 (Gibco; Thermo Fisher Scientific, Inc., Waltham, MA, USA), supplemented with $10 \%$ fetal bovine serum (Gibco; Thermo Fisher Scientific, Inc.), 100 units/ml penicillin and $100 \mu \mathrm{g} / \mathrm{ml}$ streptomycin. The cultures were maintained at $37^{\circ} \mathrm{C}$ in a humidified atmosphere with $5 \% \mathrm{CO}_{2}$ in air. The cells were seeded into a 6 -well plate and cultured for $24 \mathrm{~h}$ prior to transfection. Then either miR-199a-3p mimics, inhibitors or the negative control (NC) were transfected into the cells at a final concentration of 50 or $100 \mathrm{nM}$ using TurboFect ${ }^{\mathrm{TM}}$ in vitro Transfection reagent (Fermentas, Burlington, Canada), according to the manufacturer's protocol. The culture medium was discarded following transfection for $12 \mathrm{~h}$ and was replaced with the fresh medium. The miR-199a-3p mimics, inhibitors and the NC were all synthesized by Shanghai GenePharma Co., Ltd. (Shanghai, China).

Bioinformatics analysis. The expression abundance of miR-199a-3p in different human tissues was analyzed by GEO Datasets (http://www.ncbi.nlm.nih.gov/gds/). Using both 'miR-199a-3p' and 'Expression profiling by array' as key words, three data sets, including GSE65626, GSE53437 and GSE14985, were selected and categorized using statistical analysis. The data of GSE65626 was derived from glioblastoma tissue and matched adjacent normal tissue. The data of GSE53437 was derived from heart failure tissue and matched adjacent normal tissue. The data from GSE14985 was from multiple cancer tissue and matched adjacent normal tissue. The potential target genes of miR-199a-3p were searched for using TargetScan 6.2 (http://www.targetscan.org/). An RNAhybrid tool (bibiserv.techfak.uni-bielefeld.de/rnahybrid) was subsequently used to analyze the minimum free energy hybridization of the miR-199a-3p and target mRNAs. RNAFold WebServer (rna.tbi.univie.ac.at/cgi-bin/RNAfold.cgi) was used to predict the secondary structure of single-stranded target mRNAs. Bioinformatics analysis of promoter sequence and potential transcription factor binding sites within the 5'-regulatory region of the selected metabolic genes was performed using the EPD database (http://epd.vital-it.ch/) and MatInspector (http://www.genomatix. de/online_help/help_matinspector/matinspector_help.html).

RNA isolation of FFPE testicular tissues and Ntera-2 cells. The total RNA from FFPE tissue specimens, including testicular tumors and normal testes, were isolated using RecoverAll ${ }^{\mathrm{TM}}$ Total Nucleic Acid Isolation kit (Ambion; Thermo Fisher Scientific), according to the manufacturer's protocol. For total RNA isolation of Ntera-2 cells, RNAiso (Takara Bio., Inc., Otsu, Japan) was used.

cDNA synthesis and quantitative polymerase chain reaction ( $q P C R$ ) for miRNA and $m R N A$ detection. PrimeScript miRNA qPCR Starter kit Ver. 2.0 (Takara Bio., Inc.) was used to detect the expression of miRNA. Briefly, $3 \mu \mathrm{g}$ total RNA was digested with DNase I (Fermentas) and used for miRNA reverse transcription by PrimeScript miRNA qPCR Starter kit Ver. 2.0 (Takara Bio., Inc.) or for mRNA reverse transcription by PrimeScript RT reagent kit with gDNA Eraser (Takara Bio., Inc.). Following this, real-time qPCR was performed using a SYBR Premix Ex Taq II kit and the MX3000 instrument (Stratagene, La Jolla, CA, USA). The thermocycling conditions were as follows: Initial denaturation, $95^{\circ} \mathrm{C}$ for $10 \mathrm{~min} ; 40$ cycles of $95^{\circ} \mathrm{C}$ for $10 \mathrm{sec}$, $60^{\circ} \mathrm{C}$ for $30 \mathrm{sec}$ and $72^{\circ} \mathrm{C}$ for $32 \mathrm{sec}$. For miRNA, has-miR-15a was used as an internal control and for mRNA, GAPDH was used as an internal control. Primer sequences were as follows: GAPDH, forward: 5'-GACCCCTTCATTGACCTCAA-3' and reverse: 5'-GCATGGACTGTGGTCATGAGT-3'. The results were quantified using the $2^{-\Delta \Delta \mathrm{Cq}}$ method (14).

Cell viability analysis by 3-4,5-dimethylthiazol-2-yl-2,5 diphenyl tetrazolium bromide (MTT) assay. Ntera-2 cells at a density of $5 \times 10^{3}$ cells/well were seeded into a 96-well plate with $100 \mu \mathrm{l}$ medium for $24 \mathrm{~h}$. When the cells reached $70 \%$ confluence, they were trasfected with NC, miR-199a-3p mimics or inhibitor as above. MTT reagent $(5 \mathrm{mg} / \mathrm{ml}$; Sigma-Aldrich, St. Louis, MO, USA) was added to each well for $0,24,72 \mathrm{~h}$. The cells were subsequently incubated for $4 \mathrm{~h}$ at $37^{\circ} \mathrm{C}$ and $150 \mu \mathrm{l}$ dimethyl sulfoxide was added to each well following the removal of the medium to dissolve the formazan products. The absorbance at a wavelength of $570 \mathrm{~nm}$ in each well was determined using an ELISA reader.

Cell migration analysis by wound healing assay. Ntera-2 cells at a density of $1 \times 10^{5}$ cells/well were seeded into a 6 -well-plate 
A

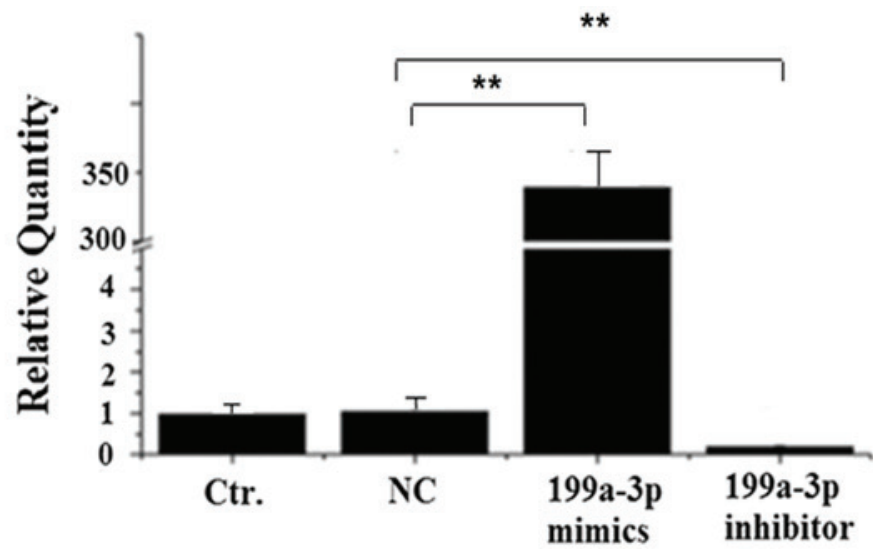

B

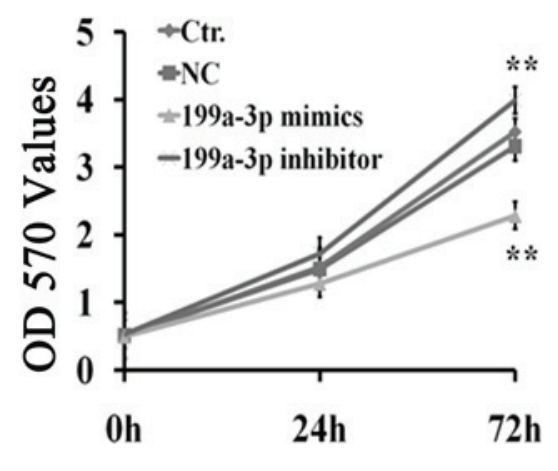

C
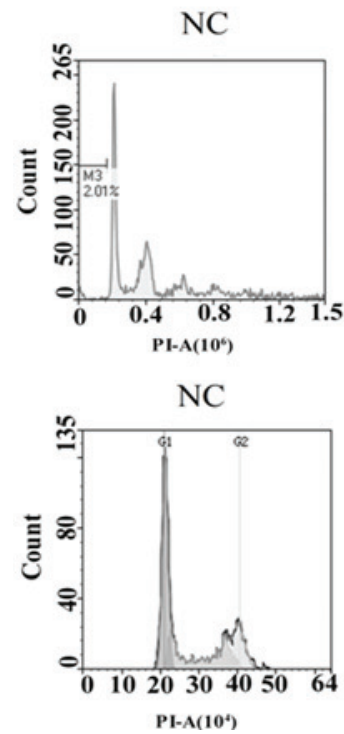

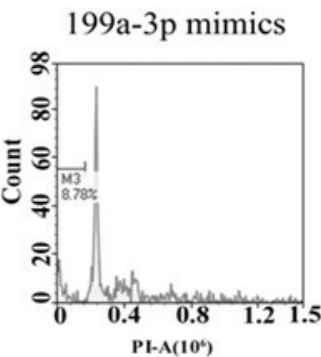

199a-3p mimics

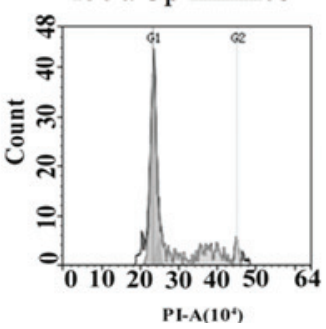

199a-3p inhibitor

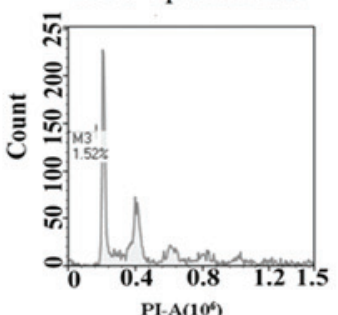

199a-3p inhibitor

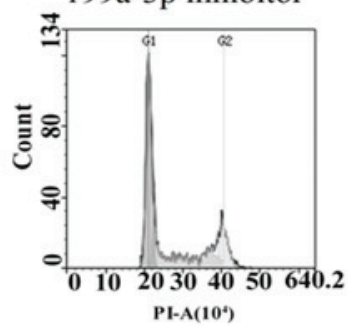

D

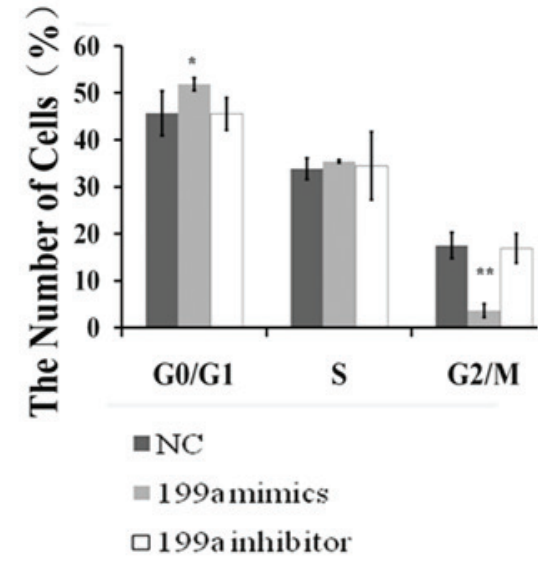

Figure 1. miR-199a-3p inhibits the proliferation of Ntera-2 cells. (A) The transfection efficiency of miR-199a-3p mimics or inhibitor after $48 \mathrm{~h}$ was assessed using reverse transcription-quantitative polymerase chain reaction. The values were normalized against that of the NC group. (B) The effects of miR-199a-3p mimics or inhibitor at 0,24 or $72 \mathrm{~h}$ on the viability of Ntera-2 cells were assessed using an 3-4,5-dimethylthiazol-2-yl-2,5 diphenyl tetrazolium bromide assay. (C and D) The effects of miR-199a-3p mimics or inhibitor on the cell cycle and apoptosis of Ntera-2 cells were analyzed by flow cytometry following staining with propidium iodide. (C) Representative cell apoptosis (above) and cell cycle distribution plots (below) and (D) the numbers of cells in each phase of the cell cycle are shown (total $\left.1 \times 10^{5}\right)$. The data are expressed as the mean \pm standard deviation $\left(\mathrm{n}=3 ;{ }^{* * *} \mathrm{P}<0.01 \mathrm{vs}\right.$. NC). miR, microRNA; OD, optical density; NC, cells transfected with scramble sequence.

with RPMI-1640 medium were grown to $80 \%$ confluence and transiently transfected with $100 \mathrm{nM} \mathrm{NC}$, miR-199a-3p mimics or inhibitors as above. The $100 \%$ confluent monolayer Ntera-2 cells were subsequently scraped with a sterile $100 \mu \mathrm{l}$ pipette tip and the cell debris was washed with D-hanks solution. The cells were incubated at $37^{\circ} \mathrm{C}$ with $5 \% \mathrm{CO}_{2}$ overnight. Images were captured under bright field light microscopy with a Nikon Eclipse E600 microscope and an RS Photometrics CoolSNAP camera at 0 and $48 \mathrm{~h}$ after scraping.

Flow cytometry analysis. For apoptosis or cell cycle analysis, $1-5 \times 10^{5}$ Ntera- 2 cells from each sample were digested with $0.25 \%$ trypsin and fixed in $70 \%$ cold ethanol overnight after resuspending in D-hanks three times. The cells were subsequently collected and were mixed with propidium iodide staining solution at a final concentration of $50 \mu \mathrm{g} / \mathrm{ml}$ for $30 \mathrm{~min}$ at room temperature in the dark. All samples were analyzed using flow cytometry, according to the manufacturer's protocols.
Glucose metabolic assay. Ntera-2 cells were transfected with either miR-199a-3p mimics or inhibitor, using $\mathrm{NC}$ as a control. The supernatants were collected after 24 and $48 \mathrm{~h}$, and detected using an Automatic Biochemical Analyzer (7170A; Hitachi, Tokyo, Japan).

qPCR array screening for metabolic genes. Briefly, the total RNA was isolated from Ntera-2 cells, which were treated with either NC, miR-199a-3p mimics or inhibitor for $48 \mathrm{~h}$. The RNA was subsequently converted into cDNA. qPCR of 148 metabolic genes was performed using a MX3000 instrument (Stratagene) by mixing equal quantities of cDNA, SYBRGreen mix (CWBio, Beijing, China) and specific primers. Each sample was analyzed in triplicate. All real-time data were normalized against GAPDH and analyzed using the $\mathrm{RT}^{2}$ profiler PCR Array Data Analysis software version 3.5 (http://pcrdataanalysis.sabiosciences. com/pcr/arrayanalysis.php). The primers used in qPCR screening are as described previously (15). 
A
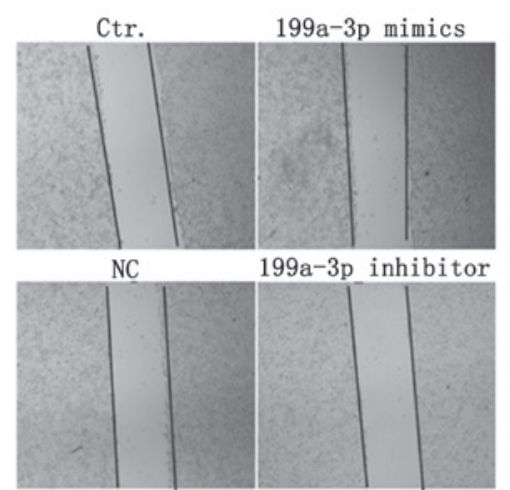

Oh

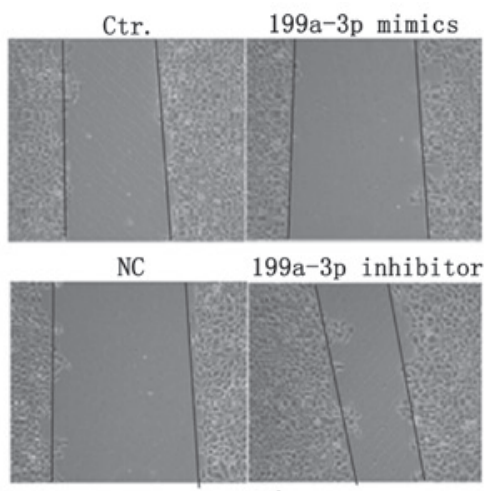

$48 \mathrm{~h}$

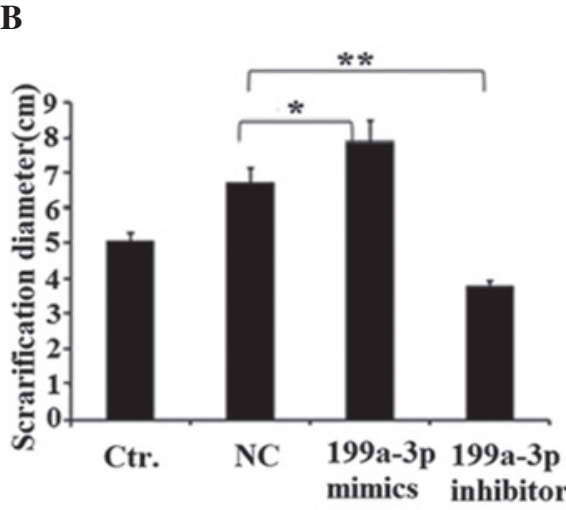

Figure 2. miR-199a-3p inhibits migration of Ntera-2 cells. (A) Ntera-2 cells were transfected with either miR-199a-3p mimics or inhibitor, using NC as control. Then cell migration was measured by wound healing assay. Images were captured at 0 and $48 \mathrm{~h}$ after Ntera- 2 cells were scraped. (B) The scarification diameter of the wound healing assay results ( $\mathrm{P}<0.05,{ }^{* * *} \mathrm{P}<0.01$ compared with the $\left.\mathrm{NC}\right)$. Ctr., control untransfected; $\mathrm{NC}$, cells transfected with scramble sequencer; miR, microRNA.

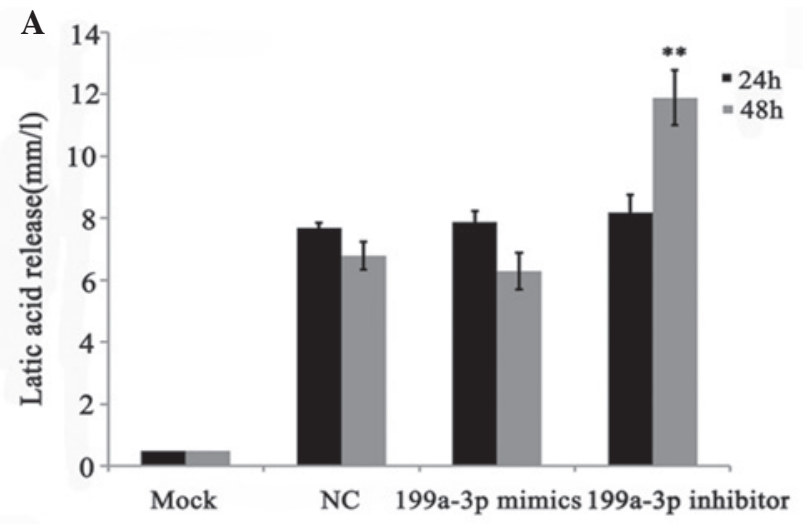

C

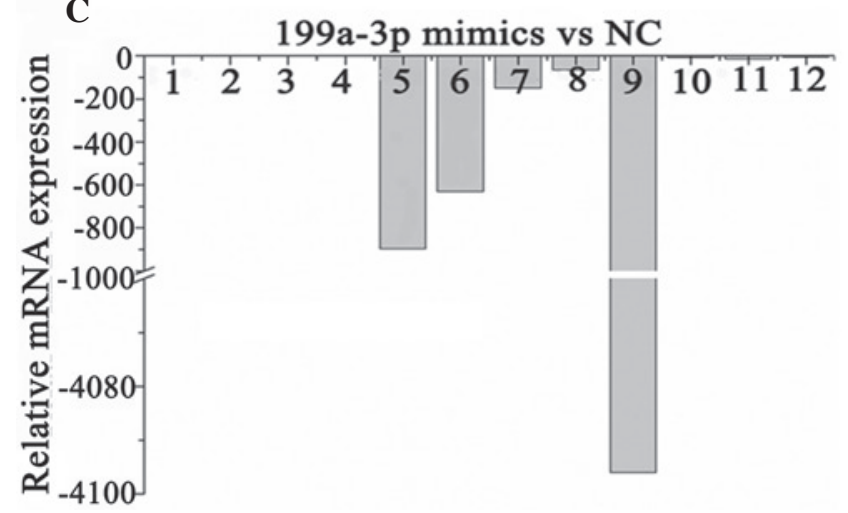

B

$199 a-3 p$ inhibitor

199a-3p mimics

NC

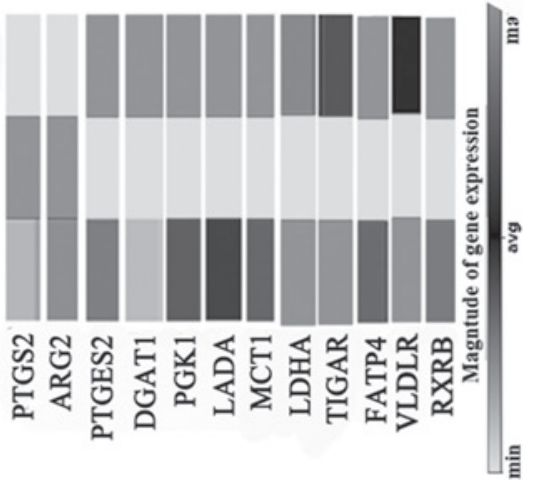

D

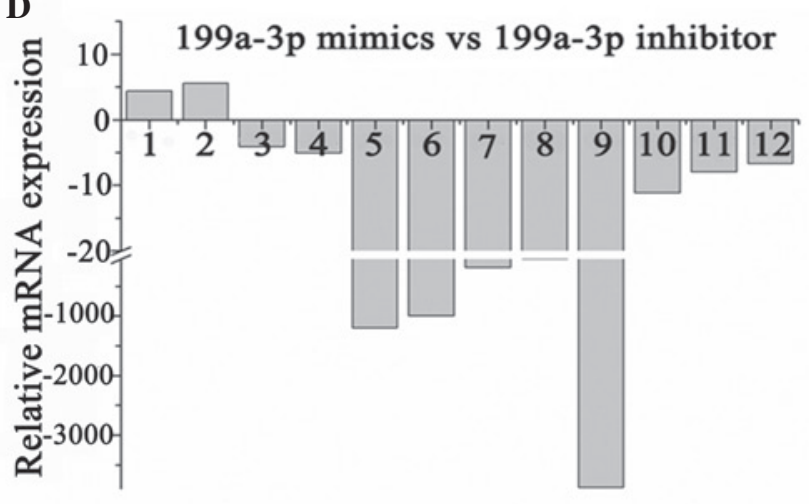

Figure 3. Impact of miR-199a-3p on the metabolism of Ntera-2 cells. (A) The production of lactate of Ntera-2 cells was detected following treatment with NC, miR-199a-3p mimics or inhibitor for 24 or 48 h. (B) A heat-map of 12 selected metabolic genes in Ntera-2 cells, which were regulated by miR-199a-3p. The expression levels are depicted by a scale from black (high expression) to gray (low expression). (C) The relative mRNA expression of 12 selected metabolic genes in the miR-199a-3p mimics group compared with the NC group. (D) The relative mRNA expression of 12 selected metabolic genes in miR-199a-3p mimics group compared with miR-199a-3p inhibitor group. ("** $\mathrm{P}<0.01)$. 1-12 refers to PTGS2, ARG2, PTGES, DGAT1, PGK1, LADA, MCT1, TIGAR, LDHA, FATP4, VLDLR and $R X R B$, respectively. NC, cells transfected with scramble sequence; PTGS2, prostaglandin-endoperoxide synthase 2; ARG2, arginase 2; PTGES, prostaglandin E synthase; DGAT1, diacylglycerol O-acyltransferase 1; PGK1, phosphoglycerate kinase 1; LADA, latent autoimmune diabetes of adults; MCT1, monocarboxylate transporter 1; TIGAR, TP53-inducible glycolysis and apoptosis regulator; LDHA, lactate dehydrogenase A; FATP4, fatty acid transport protein 4; VLDLR, very-low-density-lipoprotein receptor; RXRB, retinoid X receptor $\beta$.

Statistical analysis. The data are expressed as the mean \pm standard deviation $(\mathrm{n}=3)$. Statistical analysis was performed using the Student's t-test. $\mathrm{P} \leq 0.05$ was considered to indicate a statistically significant difference. For the qPCR array assay, the fold changes of $\geq 4$ or $\leq 0.5(\mathrm{P} \leq 0.05)$ were analyzed using t-test and P-value.

\section{Results}

miR-199a-3p inhibits the proliferation of Ntera-2 cells. Ntera-2 cells were transfected with either miR-199a-3p mimics or inhibitor, and the results of miRNA PCR confirmed that the transfection was highly efficient, stable and useful for the 


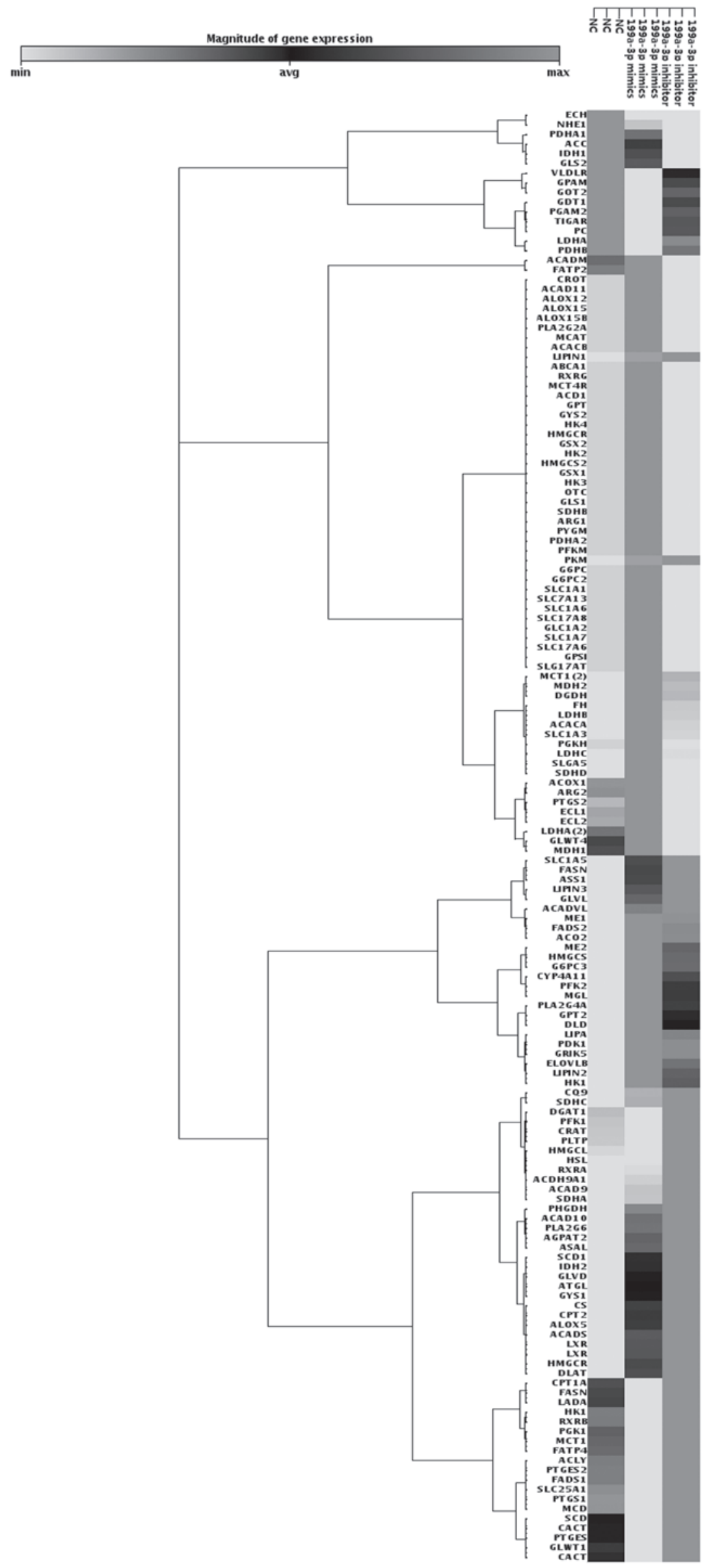

Figure 4. Impact of miR-199a-3p on the metabolism of Ntera-2 cells. A heat-map for metabolic gene profile of the Ntera-2 cells induced by miR-199a-3p was performed by quantitative polymerase chain reaction array screening. miR, microRNA; NC, cells transfected with scramble sequence. 
A

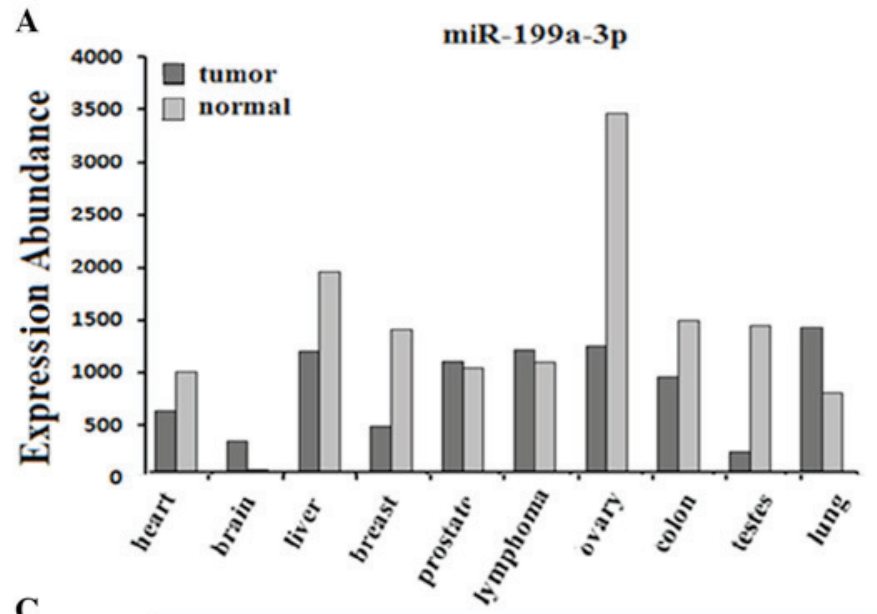

C
B

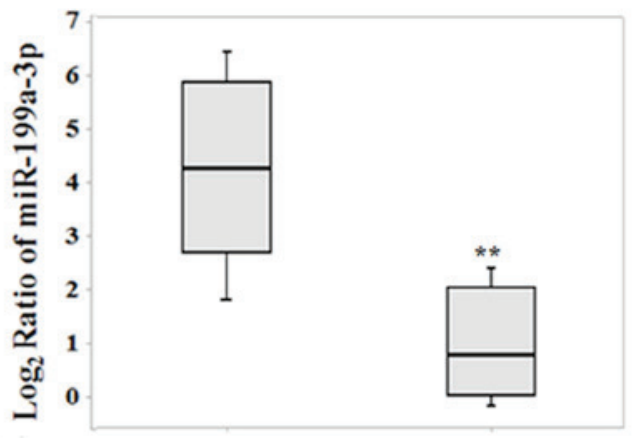

Normal testis Testicular tumor

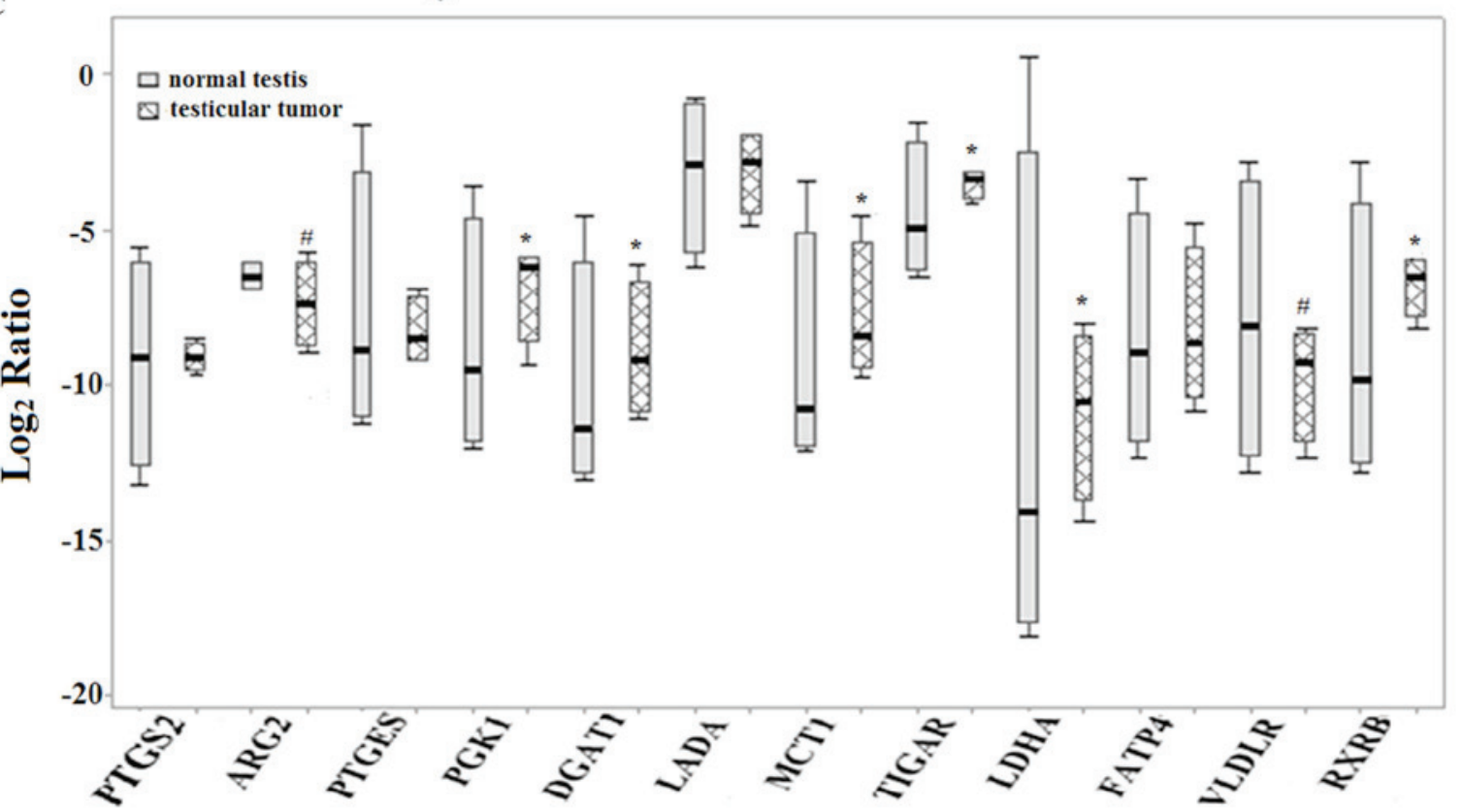

Figure 5. Expression levels of 12 selected metabolic genes in clinical testicular samples. (A) The expression profile of miR-199a-3p in various tissues was analyzed by bioinformatics. (B) The expression of miR-199a-3p in both normal testes and testicular tumors. $\left({ }^{* *} \mathrm{P}<0.01\right)(\mathrm{C})$ The expression of 12 selected genes in normal testes and testicular tumors. For clinical tissue specimens, the mRNA expressions were represented with the box plots by calculating the $\log _{2}$ ratio, which was normalized against glyceraldehyde 3-phosphate dehyrogenase $\left({ }^{*} \mathrm{P}<0.05\right.$ for upregulation, ${ }^{\#} \mathrm{P}<0.05$ for downregulation). PTGS2, prostaglandin-endoperoxide synthase 2; ARG2, arginase 2; PTGES, prostaglandin E synthase; DGAT1, diacylglycerol O-acyltransferase 1; PGK1, phosphoglycerate kinase 1; LADA, latent autoimmune diabetes of adults; MCT1, monocarboxylate transporter 1; TIGAR, TP53-inducible glycolysis and apoptosis regulator; LDHA, lactate dehydrogenase A; FATP4, fatty acid transport protein 4; VLDLR, very-low-density-lipoprotein receptor; RXRB, retinoid X receptor $\beta$.

following experiments (Fig. 1A). An MTT assay revealed that the viability and growth of Ntera- 2 cells were significantly inhibited by miR-199a-3p mimics compared with the negative control. Conversely the proliferation of cells was notably induced following treatments with the miR-199a-3p inhibitor (Fig. 1B). Using flow cytometry analysis, cell apoptosis and cell cycle distribution of Ntera-2 cells treated with NC, miR-199a-3p mimics or inhibitor were also assessed. As shown in Fig. 1C and $\mathrm{D}$, compared with the cell population of the $\mathrm{NC}$ group

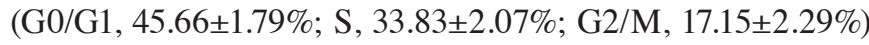
and the miR-199a-3p inhibitor group (G0/G1, 45.53 $\pm 1.46 \%$; S, 34.44 $\pm 3.76 \%$; G2/M, 16.92 $\pm 2.54 \%$ ), the miR-199a-3p mimics group exhibited an increased $\mathrm{G} 0 / \mathrm{G} 1$ phase cell population $(51.83 \pm 3.25 \%)$ and decreased $\mathrm{G} 2 / \mathrm{M}$ phase cell population $(3.61 \pm 3.15 \%)$, but no obvious effect on the percentage of $\mathrm{S}$ phase cells $(35.4 \pm 1.34 \%)$. In addition, the apoptotic cell population (pre-G1 peak) was notably increased in the miR-199a-3p mimics group $(8.78 \%)$, but not in the other groups. Therefore, these data suggested that miR-199a-3p has an anti-proliferation effect on Ntera- 2 cells and it may induce Ntera-2 cells apoptosis.

miR-199a-3p inhibits the migration of Ntera-2 cells. Cell migration was measured using a wound healing assay in Ntera-2 cells treated with NC, miR-199a-3p mimics or inhibitor. The cells treated with miR-199a-3p mimics were less migratory compared with the $\mathrm{NC}$ control or untreated cells at $48 \mathrm{~h}$ after scratching (Fig. 2A and B). By contrast, inhibition of miR-199a-3p significantly promoted the migration of Ntera- 2 cells $(\mathrm{P}<0.01$; Fig. $2 \mathrm{~B})$. This result revealed that miR-199a-3p may inhibit the migration of Ntera- 2 cells. 

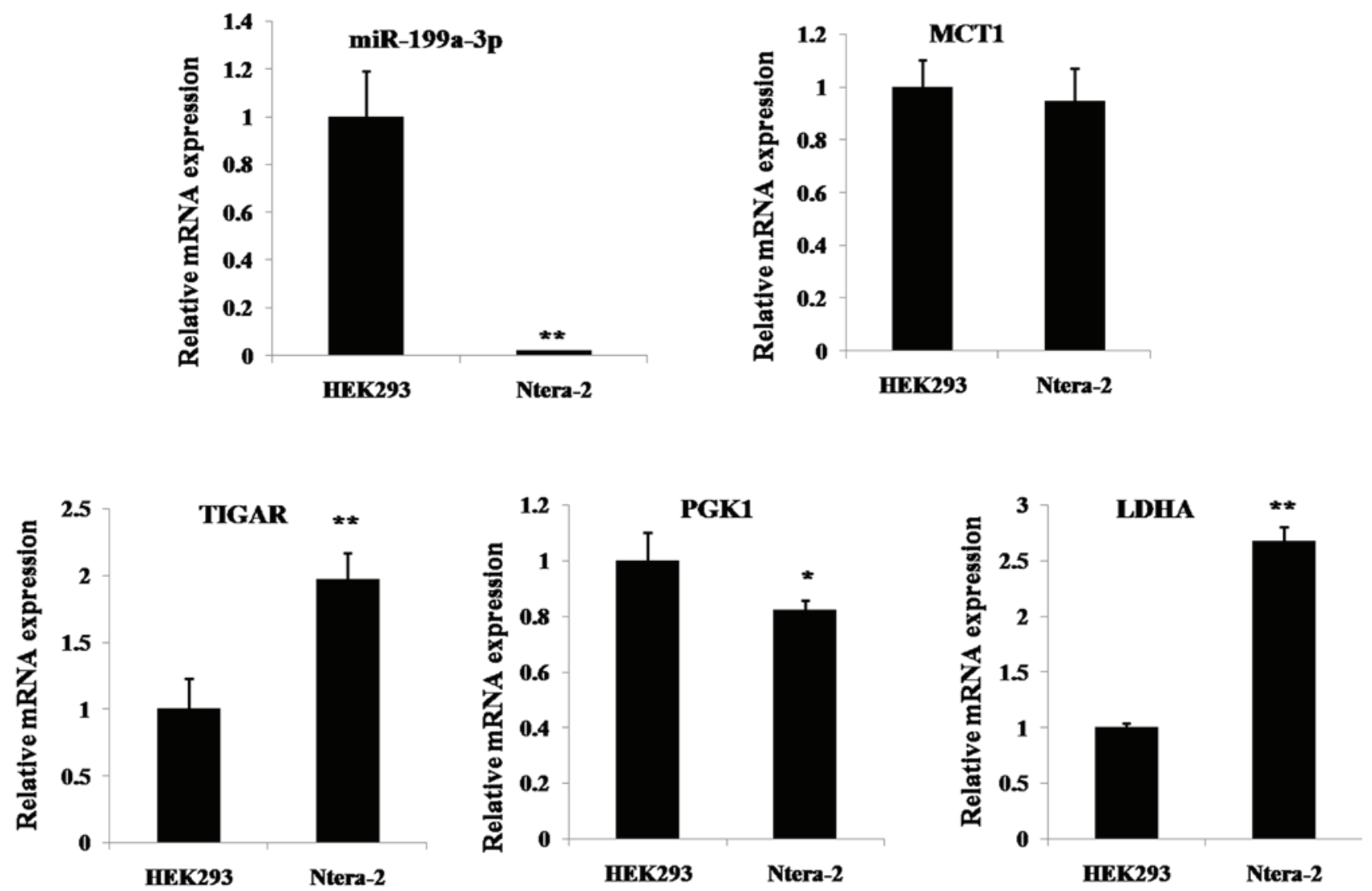

Figure 6. mRNA expression levels of miR-199a-3p and four selected metabolic genes in Ntera-2 cells and HEK293 cells. The mRNA expression levels of the selected genes were determined by reverse transcription-quantiitative polymerase chain reaction. " $\mathrm{P}<0.05,{ }^{* *} \mathrm{P}<0.01$ vs. the HEK293 cells. MCT1, monocarboxylate transporter 1; TIGAR, TP53-inducible glycolysis and apoptosis regulator; LDHA, lactate dehydrogenase A; PGK1, phosphoglycerate kinase 1.

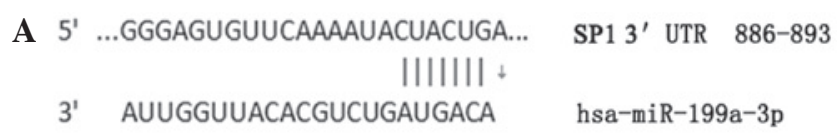

B

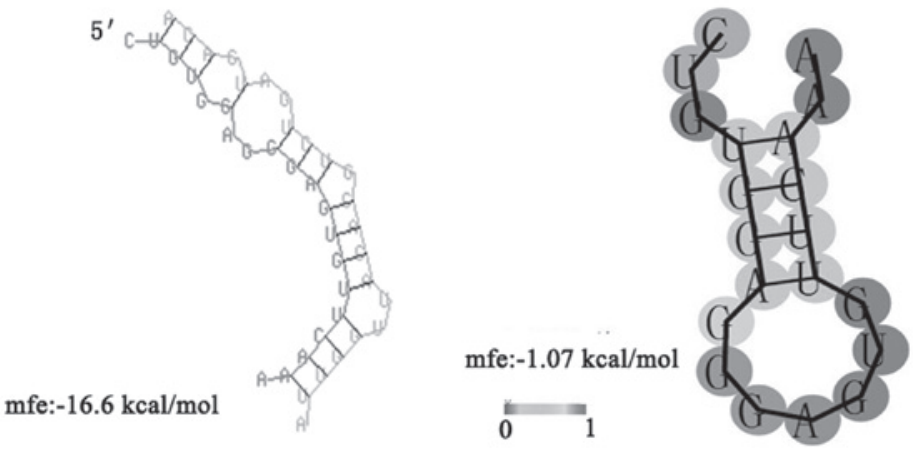

Figure 7. miR-199a-3p and its predicted target gene Sp1. (A) The predicted miR199a-3p binding sites in 3'UTR of Sp1 mRNA sequence. (B) The analysis of minimum free energy hybridization. The figure on the left shows the minimum free energy hybridization of miR199a-3p and Sp1 mRNA was predicted by RNAhybrid; the figure on the right shows the secondary structure of single-stranded Sp1 mRNAs was predicted by the RNAFold WebServer. UTR, untranslated region; miR, microRNA.

Inhibition of miR-199a-3p significantly increases the production of lactate in Ntera-2 cells. The production of lactate is an important index of glycolysis. By means of detecting lactate release of Ntera-2 cells after miR-199a-3p treatment, it was revealed that no statistically significant changes occurred in the miR-199a-3p mimics group for both 24 and $48 \mathrm{~h}$ treatment compared with the NC group (Fig. 3A). Additionally, the miR-199a-3p inhibitor group notably increased the production of lactate at $48 \mathrm{~h}$ $(\mathrm{P}<0.01)$. These results indicated that inhibition of miR-199a-3p significantly enhanced the metabolism of glucose. 
Table I. Expression of miR-199a-3p and 12 selected genes in Ntera- 2 cells transfected with the miR-199a-3p mimics or inhibitor and in testicular tumors $(n=5)$ vs. normal clinical samples $(n=5)$.

\begin{tabular}{lcc}
\hline & $\begin{array}{c}\text { miR-199a-3p } \\
\text { mimics vs. inhibitor } \\
\text { in Ntera-2 cells }\end{array}$ & $\begin{array}{c}\text { Testicular tumors } \\
\text { vs. normal testes }\end{array}$ \\
\hline miR-199a-3p & $\uparrow$ & $\downarrow$ \\
PTGS2 & $\uparrow$ & No change \\
ARG2 & $\uparrow$ & $\downarrow$ \\
PTGES & $\downarrow$ & No change \\
PGK1 & $\downarrow$ & $\uparrow$ \\
DGAT1 & $\downarrow$ & $\uparrow$ \\
LADA & $\downarrow$ & No change \\
MCT1 & $\downarrow$ & $\uparrow$ \\
TIGAR & $\downarrow$ & $\uparrow$ \\
LDHA & $\downarrow$ & $\uparrow$ \\
FATP4 & $\downarrow$ & No change \\
VLDLR & $\downarrow$ & $\downarrow$ \\
RXRB & $\downarrow$ & $\uparrow$ \\
\hline
\end{tabular}

$\uparrow$, upregulation; $\downarrow$, downregulation; miR, microRNA; PTGS2, prostaglandin-endoperoxide synthase 2; ARG2, arginase 2; PTGES, prostaglandin E synthase; DGAT1, diacylglycerolO-acyltransferase 1; PGK1, phosphoglycerate kinase 1; LADA, latent autoimmune diabetes of adults; MCT1, monocarboxylate transporter 1; TIGAR, TP53-inducible glycolysis and apoptosis regulator; LDHA, lactate dehydrogenase A; FATP4, fatty acid transport protein 4; VLDLR, very-low-density-lipoprotein receptor; $\mathrm{RXRB}$, retinoid X receptor $\beta$.

Regulation of miR-199a-3p on the metabolic gene expression profile of Ntera-2 cells. qPCR array screening was applied to investigate the changes at the mRNA level in Ntera- 2 cells following miR-199a-3p transfection by comparison with the control group (Figs. 3 and 4). Global gene expression levels induced by miR-199a-3p in Ntera- 2 cells from the qPCR array analysis are presented in Fig. 3B and Fig. 4. The differentially expressed genes with fold changes of $\geq 4$ or $\leq 0.5(\mathrm{P} \leq 0.05)$ were analyzed using t-test and P-value. As shown in Fig. 3D and $\mathrm{E}$, the expression of 12/148 genes were altered following treatment with the miR-199a-3p mimics compared with the inhibitor treatment. Of these, two genes, prostaglandin-endoperoxide synthase 2 (PTGS2) and arginase 2 (ARG2) were upregulated. The other 10 genes, prostaglandin $E$ synthase (PTGES), diacylglycerol O-acyltransferase 1 (DGAT1), PGK1, latent autoimmune diabetes of adults (LADA), MCT1, TIGAR, LDHA, fatty acid transport protein 4 (FATP4), very-low-density-lipoprotein receptor $(V L D L R)$ and retinoid $X$ receptor $\beta(R X R B)$ were downregulated specifically. Whereas, eight downregulated genes (PGK1, LADA, MCT1, TIGAR, LDHA, FATP4, VLDLR, RXRB) were observed in miR-199a-3p mimics group compared with the NC group.

Expression abundance of miR-199a-3p and selected metabolic genes in both TGCT tissues and cell lines. The clinical relevance of aberrant metabolism caused by dysregulated
miR-199a-3p signaling was determined using TGCTs and normal testis tissue specimens. In accordance with the bioinformatics analysis (Fig. 5A), the miR-199a-3p expression in testicular tumors was obviously decreased compared with that in normal testes. For the selected genes, PTGS2, PTGES, $L A D A$ and FATP4 revealed no significant change between normal testes and testicular tumors, while $A R G 2$ and $V L D L R$ were downregulated in the clinical samples (Fig. 5C; Table I). Notably, the other four genes, including LDHA, MCT1, PGK1 and TIGAR, were markedly upregulated in testicular tumors, which were similar to the results in Ntera-2 cells. In order to further understand the association between miR-199a-3p and the four selected metabolic genes, LDHA, MCT1, PGK1 and TIGAR, the expression levels in cell lines, including HEK 293 and Ntera-2 cells were detected. Fig. 6 demonstrated that miR-199a-3p is expressed at a higher level in HEK293 cells compared with the levels in Ntera-2 cells. For the four selected genes, the mRNA level of MCT1 was similar in both HEK293 and Ntera-2 cells, while the mRNA expression of PGK1 was slightly higher in HEK293 cells compared with in Ntera-2 cells. By contrast, a significantly lower expression of both TIGAR and LDHA was present in HEK293 cells compared with the Ntera-2 cells, which is the opposite expression signature compared with miR-199a-3p.

miR-199a-3p may downregulate the expression of four metabolic genes via the Spl binding site. Using TargetScan analysis, it was demonstrated that none of the four selected genes (LDHA, MCT1, PGK1 and TIGAR) have the potential recognition site of miR-199a-3p, which suggests that the regulation is not direct. In order to understand how miR-199a-3p downregulates the expression of these genes, the promoter sequence of the four genes was analyzed and revealed that the binding site of Sp1, a common transcription factor, was in the promotor region of all four selected genes (data not shown). Notably, it was predicted using TargetScan analysis that miR-199a-3p binds to the target sequences in the 3'-untranslated region (UTR) of Sp1 mRNA. In addition, further bioinformatics analysis revealed that the minimum free energy hybridization of miR-199a-3p binding with the target gene Sp1 3'UTR was markedly lower compared with that of the secondary structure of single-stranded Sp1 mRNA, indicating that Sp1 and miR-199a-3p have a higher possibility for binding (Fig. 7). Therefore, the present study hypothesized that miR-199a-3p may downregulate the four metabolic genes via Sp1.

\section{Discussion}

The metabolic shift to aerobic glycolysis is a common hallmark of cancer. Normally, non-cancerous cells catabolize glucose by oxidative phosphorylation in the mitochondria to produce adenosine triphosphate (ATP). However, in proliferating cancer cells, glucose carbons were predominantly converted to lactate, even with supply of adequate oxygen. This phenomenon of metabolic alteration in cancer is termed the 'Warburg effect' (16). Advances in cancer metabolism research over the last decade have demonstrated that metabolic alterations can enhance a cancer cells capability for proliferation, migration and invasion. In addition, clinical studies have shown a close correlation between elevated levels of lactate and poor patient prognosis or 
overall survival in different cancer types (17). Therefore, certain biological molecules involved in metabolic process are currently being considered as therapeutic targets for cancer.

Since it was discovered that miRNAs are abnormally expressed in cancer, increasing data has revealed that miRNAs serve crucial roles in tumor growth by regulating their target genes and a large number of miRNAs have been identified to regulate cancer metabolism (18). Previous studies showed that the alterations of miR-199a-3p, a member of miR-199a family that controls the fate of cell survival and death, were associated with the pathogenesis and progression of cancer, and it can behave either as an oncogene or as tumor suppressor in different cancer types (19-21). In the present study, bioinformatics analysis (Fig. 5A) revealed that the expression of miR-199a-3p was upregulated in tumors, including brain and lung cancer, and downregulated in tumors, including liver, breast, ovary, colon and testicular cancer, suggesting that patterns of miR-199a-3p expression tend to vary among tumor types and its functions are complicated in different tissues. The present study also investigated that overexpression of miR-199a-3p caused the inhibition of Ntera- 2 cell proliferation and apoptosis, G1 and G2 phase arrest and suppressed cell migration (Figs. 1 and 2), which is consistent with a previous study and suggested miR-199a-3p was a tumor suppressor in testicular tumor cells.

To improve the understanding of the functional mechanism of miR-199a-3p as a tumor suppressor in testicular cancer, the present study performed a biochemical method and high-throughput qPCR array screening analysis to detect the effect of miR-199a-3p on global glycolysis metabolism pattern in Netra-2 cells. As shown in Figs. 3 and 4, the inhibition of miR-199a-3p increased the production of lactate and a list of metabolic genes regulated by miR-199a-3p was identified. Of these, two genes (PTGS2, ARG2) were upregulated and 10 genes (PTGES, DGAT1, PGK1, LADA, MCT1, TIGAR, $L D H A, F A T P 4, V L D L R, R X R B)$ were downregulated specifically. In clinical samples, the expression of miR-199a-3p was clearly reduced in testicular germ cell tumors compared with normal testicular tissue. The selected metabolic genes, including LDHA, MCT1, PGK1 and TIGAR, downregulated by miR-199a-3p in Netera-2 cells, were significantly overexpressed in malignant testicular tumors, and the expression of these genes was inversely correlated with the expression of miR-199a-3p (Fig. 5; Table I). Among these four genes, a significantly lower expression of both TIGAR and LDHA was present in human embryonic kidney cells (HEK293) compared with testicular tumor cells (Ntera-2), which is the opposite expression signature compared with miR-199a-3p (Fig. 6). These results suggested that miR-199a-3p may serve important roles in aerobic glycolysis and tumorigenesis.

During aerobic glycolysis, the majority of the pyruvate is converted into lactate in the cytoplasm by enzyme lactate dehydrogenase (LDH). Lactate is subsequently secreted outside of the cells by MCT1. Overexpression of MCT1 and LDHA (one of the predominant LDH isoforms) has been reported in a variety of solid tumor types, including colorectal, melanoma, breast and pancreatic cancer (22-25). In order to determine what may influence the expression levels of MCT1 or LDHA, several previous studies have focused on examining certain factors, including hypoxia, p53 status and chemotherapeutics (26,27). Previously, a few studies have focused on the association between glucose metabolism and miRNA regulation in tumors. For instance, miR-124 is a direct regulator of MCT1 in medulloblastoma cells and miR-375 is involved in regulation of lactate dehydrogenase $\mathrm{B}$ (LDHB). The present study indicated that both $L D H A$ and MCT1 may be negatively regulated by miR-199a-3p in testicular germ cell tumors.

The other selected gene in this metabolic qPCR array screening is $P G K 1$, one of the major glycolytic enzymes, which catalyzes the conversion of 1,3-diphosphoglycerate to 3-phosphoglycerate. PGK1 has been previously demonstrated to be associated with the HER-2/neu status in breast cancer (28) and a poor outcome for patients who have lung adenocarcinoma and who are multi-drug resistant $(29,30)$, suggesting its potential as a biomarker for cancer. In lung cancer cell lines, PGK1 was revealed to be regulated by miR-29a and the present study has linked its expression to miR199a-3p in tumor biology.

However, these four selected genes ( $L D H A, M C T 1, P G K 1$ and TIGAR) lack the potential recognition sites of miR-199a-3p, meaning the regulation of miR-199a-3p on four genes is not direct. Notably, the present study noticed that the potential binding site of $\mathrm{Sp} 1$ was present in the promoter regions of all four selected genes. In addition, it was predicted that miR-199a-3p can bind to target sequences in the 3'-UTR of Sp1 mRNA by using TargetScan analysis (Fig. 7). Sp1, a member of Sp/Kruppel super family, is a ubiquitous transcription factor that serves an important role in controlling the transcription of numerous genes that contain GC boxes in their promoters, particularly to those genes associated with the metabolism of nucleic acids and biosynthesis $(31,32)$. Abnormal activation of Sp1 occurs in a wide variety of human tumor types and high levels of Sp1 protein are considered a negative prognostic factor $(33,34)$. The present study speculated that miR-199a-3p may downregulate the four metabolic genes through Sp1.

In conclusion, the present study characterized the role of miR-199a-3p in regulating the metabolism of Ntera-2 cells. These results suggested that miR-199a-3p is a major metabolic regulator in tumor suppression and miR-199a-3p may downregulate metabolic genes (LDHA, PGK1, MCT1, TIGAR) through the transcription factor Sp1. This led to the speculation that downregulation of miR-199a-3p may be a potential cause of overexpression of these metabolic genes and targeting miR-199a-3p may be an innovative strategy in cancer treatment.

\section{Acknowledgements}

The present study was supported by the National Natural Science Foundation of China (nos. 81270735 and 81372182).

\section{References}

1. Bartel DP: MicroRNAs: Genomics, biogenesis, mechanism and function. Cell 116: 281-297, 2004.

2. Lee RC, Feinbaum RL and Ambros V: The C. elegans heterochronic gene lin-4 encoded small RNAs with antisense complementarity to lin-14. Cell 75: 843-854, 1993.

3. Lewis BP, Burge CB and Bartel DP: Conserved seed pairing, often flanked by adenosines, indicates that thousands of human genes are microRNA targets. Cell 120: 15-20, 2005.

4. Huang Y, Shen XJ, Zou Q, Wang SP, Tang SM and Zhang GZ: Biological functions of microRNAs: A review. J Physiol Biochem 67: 129-139, 2011.

5. Di Leva G and Croce CM: miRNA profiling of cancer. Curr Opin Genet Dev 23: 3-111, 2013. 
6. Zhang B, Pan X, Cobb GP and Anderson TA: MicroRNAs as oncogenes and tumor suppressors. Dev Biol 302: 1-12, 2007.

7. Garzon R, Calin GA and Croce CM: MicroRNAs in cancer. Annu Rev Med 60: 167-179, 2009.

8. Di Leva G and Croce CM: Roles of small RNAs in tumor formation. Trends Mol Med 16: 257-267, 2010.

9. Bezan A, Gerger A and Pichler M: MicroRNAs in testicular cancer: Implications for pathogenesis, diagnosis, prognosis and therapy. Anticancer Res 34: 2709-2713, 2014

10. Voorhoeve PM, le Sage C, Schrier M, Gillis AJ, Stoop H, Nagel R, Liu YP, van Duijse J, Drost J, Griekspoor A, et al: A genetic screen implicates miRNA-372 and miRNA-373 as oncogenes in testicular germ cell tumors. Cell 124: 1169-1181, 2006.

11. Chen BF, Gu S, Suen YK, Li L and Chan WY: microRNA-199a-3p, DNMT3A, and aberrant DNA methylation in testicular cancer. Epigenetics 9: 119-128, 2014

12. Rong Z, Li D, Liu X, Liu Z, Wu D and Liu X: Screening for miRNAs and their potential targets in response to TGF- $\beta 1$ based on miRNA microarray and comparative proteomics analyses in a mouse GC-1 spg germ cell line. Int J Mol Med 35: 821-828, 2015

13. Zhang Y, Fan KJ, Sun Q, Chen AZ, Shen WL, Zhao ZH, Zheng XF and Yang $X$ : Functional screening for miRNAs targeting Smad4 identified miR-199a as a negative regulator of TGF- $\beta$ signalling pathway. Nucleic Acids Res 40: 9286-9297, 2012.

14. Livak KJ and Schmittgen TD: Analysis of relative gene expression data using real-time quantitative PCR and the 2(-Delta Delta C(T)) method. Methods 25: 402-408, 2001.

15. Xiao L, Hu Z, Dong X, Tan Z, Li W, Tang M, Chen L, Yang L, Tao Y, Jiang Y, et al: Targeting epstein-barr virus oncoprotein LMP1-mediated glycolysis sensitizes nasopharyngeal carcinoma to radiation therapy. Oncogene 33: 4568-4578, 2014.

16. Soga T: Cancer metabolism: Key players in metabolic reprogramming. Cancer Sci 104: 275-281, 2013.

17. Han T, Kang D, Ji D, Wang X, Zhan W, Fu M, Xin HB and Wang JB: How does cancer cell metabolism affect tumor migration and invasion? Cell Adh Migr 7: 395-403, 2013.

18. Chan B, Manley J, Lee J and Singh SR: The emerging roles of microRNAs in cancer metabolism. Cancer Lett 356: 301-308, 2015

19. Brenner B, Hoshen MB, Purim O, David MB, Ashkenazi K, Marshak G, Kundel Y, Brenner R, Morgenstern S, Halpern M, et al:MicroRNAs as a potential prognostic factor in gastric cancer. World J Gastroenterol 17: 3976-3985, 2011.

20. Wan D, He S, Xie B, Xu G, Gu W, Shen C, Hu Y, Wang X, Zhi Q and Wang L: Aberrant expression of miR-199a-3p and its clinical significance in colorectal cancers. Med Oncol 30: 378, 2013.

21. Feber A, Xi L, Pennathur A, Gooding WE, Bandla S, Wu M, Luketich JD, Godfrey TE and Litle VR: MicroRNA prognostic signature for nodal metastases and survival in esophageal adenocarcinoma. Ann Thorac Surg 91: 1523-1530, 2011.
22. Le Floch R, Chiche J, Marchiq I, Naiken T, Ilc K, Murray CM Critchlow SE, Roux D, Simon MP nd Pouysségur J: CD147 subunit of lactate/H+ symporters MCT1 and hypoxia-inducible MCT4 is critical for energetics and growth of glycolytic tumors. Proc Natl Acad Sci USA 108: 16663-16668, 2011.

23. Pinheiro C, Albergaria A, Paredes J, Sousa B, Dufloth R, Vieira D, Schmitt F and Baltazar F: Monocarboxylate transporter 1 is up-regulated in basal-like breast carcinoma. Histopathology 56: 860-867, 2010

24. Miranda-Gonçalves V, Honavar M, Pinheiro C, Martinho O, Pires MM, Pinheiro C, Cordeiro M, Bebiano G, Costa P, Palmeirim I, et al: Monocarboxylate transporters (MCTs) in gliomas: Expression and exploitation as therapeutic targets. Neuro Oncol 15: 172-188, 2013.

25. Pinheiro C, Longatto-Filho A, Azevedo-Silva J, Casal M, Schmitt FC and Baltazar F: Role of monocarboxylate transporters in human cancers: State of the art. J Bioenerg Biomembr 44: 127-139, 2012.

26. Walters DK, Arendt BK and Jelinek DF: CD147 regulates the expression of MCT1 and lactate export in multiple myeloma cells. Cell Cycle 12: 3175-3183, 2013.

27. Feng L, E LL, Soloveiv MM, Wang DS, Zhang B, Dong YW and Liu HC: Synergistic cytotoxicity of cisplatin and Taxol in overcoming Taxol resistance through the inhibition of LDHA in oral squamous cell carcinoma. Oncol Lett 9: 1827-1832, 2015.

28. Zhang D, Tai LK, Wong LL, Chiu LL, Sethi SK and Koay ES: Proteomic study reveals that proteins involved in metabolic and detoxification pathways are highly expressed in her-2/neu-positive breast cancer. Mol Cell Proteomics 4: 1686-1696, 2005.

29. Chen G, Gharib TG, Wang H, Huang CC, Kuick R, Thomas DG, Shedden KA, Misek DE, Taylor JM, Giordano TJ, et al: Protein profiles associated with survival in lung adenocarcinoma. Proc Natl Acad Sci USA 100: 13537-13542, 2003.

30. Duan Z, Lamendola D, Yusuf R, Penson R, Preffer F and Seiden M: Overexpression of human phosphoglycerate kinase 1 (PGK1) induces a multidrug resistance phenotype. Anticancer Res 22: 1933-1941, 2002

31. Li L, He S, Sun JM and Davie JR: Gene regulation by Sp1 and Sp3. Biochem Cell Biol 82: 460-471, 2004.

32. Solomon SS, Majumdar G, Martinez-Hernandez A and Raghow R: A critical role of Sp1 transcription factor in regulating gene expression in response to insulin and other hormones. Life Sci 83: 305-312, 2008

33. Safe $\mathrm{S}$ and Abdelrahim M: Sp transcription factor family and its role in cancer. Eur J Cancer 41: 2438-2448, 2005.

34. Vizcaíno C, Mansilla S and Portugal J: Sp1 transcription factor: A long-standing target in cancer chemotherapy. Pharmacol Ther 152: 111-124, 2015. 